\title{
2-18F-Fluoroethanol Is a PET Reporter of Solid Tumor Perfusion
}

\author{
Brennan J. Wadsworth ${ }^{1,2}$, Jinhe Pan ${ }^{3}$, Iulia Dude ${ }^{3}$, Nadine Colpo ${ }^{3}$, Momir Bosiljcic ${ }^{1,2}$, Kuo-Shyan Lin*3, \\ Francois Benard*3, and Kevin L Bennewith*1,2 \\ ${ }^{I}$ Integrative Oncology, BC Cancer Agency, Vancouver, British Columbia, Canada; ${ }^{2}$ Pathology and Laboratory Medicine, University of \\ British Columbia, Vancouver, British Columbia, Canada; and ${ }^{3}$ Molecular Oncology, BC Cancer Agency, Vancouver, British \\ Columbia, Canada
}

Solid tumor perfusion is a proven variable of interest for predicting cancer aggression and response to therapy. Current methods for noninvasively imaging tumor perfusion with PET are limited by restricted accessibility and short half-lives of perfusion radiotracers. This study presents $2-{ }^{18} \mathrm{~F}$-fluoroethanol $\left(2-{ }^{18} \mathrm{~F}-\mathrm{FEtOH}\right)$ as a perfusion reporter that can distinguish between tumors of varying perfusion levels and can be applied to screening drugs that modify tumor perfusion. Methods: Uptake of ${ }^{2-}{ }^{18} \mathrm{~F}-\mathrm{FEtOH}$ in $4 \mathrm{~T} 1$ and $67 \mathrm{NR}$ murine mammary carcinoma tumors grown in mice was measured using ex vivo radiography as well as static and dynamic PET imaging. $2-{ }^{18} \mathrm{~F}-\mathrm{FEtOH}$ uptake was directly compared with the ${ }^{14} \mathrm{C}$ iodoantipyrine perfusion reporter, and the perfusion-modifying drugs nicotinamide, pentoxifylline, and hydralazine were used to manipulate tumor perfusion before $2-{ }^{18} \mathrm{~F}-\mathrm{FEtOH}$ quantification. Results: Uptake of $2-{ }^{18} \mathrm{~F}-\mathrm{FEtOH}$ in $4 \mathrm{~T} 1$ and $67 \mathrm{NR}$ tumors was consistent with known perfusion differences within and between these tumors. $2-{ }^{18} \mathrm{~F}-\mathrm{FEtOH}$ uptake corresponded well with ${ }^{14} \mathrm{C}$-iodoantipyrine and reflected the tumor perfusion-modifying effects of each drug. Conclusion: $2-{ }^{18} \mathrm{~F}-$ $\mathrm{FEtOH}$ is a novel ${ }^{18} \mathrm{~F}$-based radiotracer for investigating tumor perfusion with PET imaging. Quantification of ${ }^{2-}{ }^{18} \mathrm{~F}-\mathrm{FEtOH}$ uptake can be used to distinguish between tumors of varying perfusion and to screen the efficacy of blood flow-modifying drugs for use as adjuvants to existing cancer therapies.

Key Words: positron emission tomography; tumor perfusion; $2-{ }^{18} \mathrm{~F}-$ fluoroethanol; hydralazine; nicotinamide

J Nucl Med 2017; 58:815-820

DOI: 10.2967/jnumed.116.183624

$\mathbf{N}$ oninvasive imaging modalities are critical components of cancer care that can visualize the varied and widespread nature of cancer, and PET is a workhorse technique for noninvasive imaging. The utility of PET has evolved from detecting metabolically active tumors with ${ }^{18} \mathrm{~F}$-FDG toward a broader ability to characterize the tumor microenvironment. Within this trend are radiotracer indicators of tumor hypoxia (i.e., regions of low oxygen content), such as ${ }^{18} \mathrm{~F}$-fluoromisonidazole, ${ }^{18} \mathrm{~F}$-fluoroazomycin arabinoside, and ${ }^{18} \mathrm{~F}-\mathrm{EF} 5$. Tumor hypoxia is associated with reduced overall

Received Sep. 9, 2016; revision accepted Dec. 21, 2016.

For correspondence or reprints contact: Kevin Bennewith, \#10-108, 675

West 10th Ave., Vancouver, British Columbia, Canada, V5Z1L3.

E-mail: kbennewi@bccrc.ca

${ }^{*}$ Contributed equally to this work.

Published online Jan. 26, 2017.

COPYRIGHT (C 2017 by the Society of Nuclear Medicine and Molecular Imaging. survival and poor prognosis in multiple cancer types (1) because hypoxic cells are resistant to therapy and exhibit multifaceted support of metastasis (2). Successes of PET hypoxia reporters in patient studies include distinguishing aggressive high-grade glioblastoma multiform from less aggressive astrocytoma and oligodendrioma (3) and predicting resistance to primary endocrine therapy in breast cancer (4).

Hypoxia develops in tumors from a combination of high metabolic demand and insufficient delivery of oxygenated blood. Tumor vasculature is typically abnormal, exhibiting low vascular density and a dysfunctional architecture prone to leakiness and regional perfusion loss (5). The fact that hypoxia develops in regions with insufficient perfusion creates a challenge for detecting tumor hypoxia via PET imaging due to concerns that the radiotracer may not have access to all hypoxic regions. Indeed, some reports in patients suggest that tumor regions with low perfusion are not always matched with high uptake of hypoxia reporters $(6,7)$ despite the high likelihood of low regional oxygen content. Tumor perfusion itself is also a primary variable of interest, because tumor perfusion influences nutrient delivery to the tumor with potential effects on tumor metabolism and progression, and tumor perfusion also affects delivery of chemotherapeutics. Measuring tumor perfusion either at baseline or before and after a therapy cycle has predicted disease-free survival in patients with breast cancer $(8,9)$, response to chemotherapy in head and neck cancer (10), response of cervical cancer to radiation therapy (11), and response of glioblastoma to antiangiogenic therapy (12). In addition to complementing hypoxia reporters and independently providing prognostic data, noninvasive imaging of tumor perfusion could be used preclinically to study strategies for modifying tumor perfusion for either radiosensitization or manipulating chemotherapy delivery.

The gold standard in PET-based imaging of perfusion is ${ }^{15} \mathrm{O}-$ $\mathrm{H}_{2} \mathrm{O}$ (half-life, $2 \mathrm{~min}$ ). The initial development of ${ }^{15} \mathrm{O}-\mathrm{H}_{2} \mathrm{O}$ was for myocardial perfusion imaging $(13,14)$ and cerebral perfusion imaging (15), although ${ }^{15} \mathrm{O}-\mathrm{H}_{2} \mathrm{O}$ has recently been used for imaging tumor perfusion (6-8). The main challenge with using ${ }^{15} \mathrm{O}$ $\mathrm{H}_{2} \mathrm{O}$ and other established PET reporters of perfusion with rapid half-lives, such as ${ }^{15} \mathrm{O}$-butanol (16) and ${ }^{13} \mathrm{~N}^{-\mathrm{NH}_{3}}$ (17) (half-life, $10 \mathrm{~min}$ ), is the requirement of an onsite cyclotron for production and nearby administration. ${ }^{82} \mathrm{Rb}$ (half-life, $1.25 \mathrm{~min}$ ) is a validated perfusion reporter (18) that can be produced using a ${ }^{82} \mathrm{Sr} /{ }^{82} \mathrm{Rb}$ generator, thus alleviating the need for an onsite cyclotron. However, ${ }^{82} \mathrm{Rb}$ suffers from a long positron range (19), which reduces PET resolution. ${ }^{82} \mathrm{Rb}$ also exhibits relatively low tissue extraction from the blood, at least in myocardium studies, which results in 
low contrast between well-perfused and poorly perfused regions (20). We believe that development of an ${ }^{18} \mathrm{~F}$ - (half-life, $110 \mathrm{~min}$ ) based reporter of tumor perfusion will provide both logistical and accessibility benefits, while possessing the shortest positron range (and therefore highest PET resolution) of the radioisotopes discussed (19).

This study presents $2{ }^{18} \mathrm{~F}$-fluoroethanol $\left(2-{ }^{18} \mathrm{~F}-\mathrm{FEtOH}\right)$ as a novel reporter of tumor perfusion. We have previously shown that $2-{ }^{18} \mathrm{~F}-\mathrm{FEtOH}$ indiscriminately labels well-perfused organs in the body, exhibits relatively low rates of defluorination, and provides a large window of time for imaging because of an extended plateau of activity that is protected from biologic clearance (21). This plateau is believed to arise from intracellular conversion of $2-{ }^{18} \mathrm{~F}-\mathrm{FEtOH}$ to ${ }^{18} \mathrm{~F}$-fluoroacetate followed by conversion to ${ }^{18} \mathrm{~F}$-fluoroacetylCoA and ${ }^{18} \mathrm{~F}$-fluorocitrate, both of which are trapped inside cells $(22)$. We predict that this $2-{ }^{18} \mathrm{~F}$ FEtOH-mediated accumulation of ${ }^{18} \mathrm{~F}$ inside well-perfused cells will enhance contrast against poorly perfused cells to provide strong resolution of intratumoural regional differences in perfusion status and allow for imaging of tumor perfusion well after the injection of the radiotracer.

We aimed to validate $2-{ }^{18} \mathrm{~F}-\mathrm{FEtOH}$ as a reporter of tumor perfusion using PET in murine tumor models. We assessed whether $2-{ }^{18} \mathrm{~F}-\mathrm{FEtOH}$ uptake would discriminate between tumors with differing perfusion phenotypes using $67 \mathrm{NR}$ and $4 \mathrm{~T} 1$ murine mammary carcinomas (23). To determine whether $2-{ }^{18} \mathrm{~F}-\mathrm{FEtOH}$ uptake is specific to perfusion status, we conducted direct comparison to the established perfusion reporter ${ }^{14} \mathrm{C}$-iodoantipyrine $\left({ }^{14} \mathrm{C}\right.$-IAP) $(24)$, we conducted kinetic analysis of dynamic PET scans to ensure $2-{ }^{18} \mathrm{~F}$ FEtOH uptake was dependent on plasma input, and we manipulated tumor perfusion with the blood flow-modifying agents nicotinamide, pentoxifylline, and hydralazine. Our data indicate that $2-{ }^{18} \mathrm{~F}-\mathrm{FEtOH}$ is a radiotracer applicable to quantifying native or drug-induced changes in tumor perfusion by PET.

\section{MATERIALS AND METHODS}

\section{2-18 F-FEtOH Synthesis}

$2-{ }^{18} \mathrm{~F}-\mathrm{FEtOH}$ was synthesized as described previously (21).

\section{${ }^{18} \mathrm{~F}$ y-Counting and ${ }^{14} \mathrm{C}$ Liquid Scintillation Counting}

${ }^{14} \mathrm{C}$-IAP and $2-{ }^{18} \mathrm{~F}-\mathrm{FEtOH}$ were coinjected into anesthetized mice, which were sacrificed $2 \mathrm{~min}$ after injection for tissue harvest. Harvested tissue was subject to $\gamma$-counting for detection of ${ }^{18} \mathrm{~F}$ activity using a Wizard2 2480 automatic $\gamma$-counter (Perkin Elmer) followed by ${ }^{14} \mathrm{C}$ counting using a RackBeta1219 liquid scintillation counter (LKB Wallac).

\section{Mouse Tumor Models}

Ten-week-old female BALB/c mice were purchased from Taconic and housed in the Animal Resource Centre at the BC Cancer Agency Research Centre under specific pathogen-free conditions. 67NR and 4T1 murine mammary carcinoma cell lines (gifts from Dr. Fred Miller, Karmanos Cancer Institutes) were maintained in RPMI 1640 medium + $10 \%$ fetal bovine serum and used within 20 passages. All animal experiments were performed in accordance with Institutional and Canadian Council on Animal Care guidelines.

\section{Antibodies}

Tumor sections were stained with unconjugated CD31 antibody (BD Pharmingen) with Alexa 594 secondary antibody (Invitrogen) and fluorescein isothiocyanate-conjugated antibody against pimonidazole (Hypoxyprobe). Pimonidazole (100 mg/kg) was injected intraperitoneally $1.5 \mathrm{~h}$ before mouse sacrifice. Images were captured with a Retiga EXi camera (QImaging) using an Axiovert S100 microscope (Carl Zeiss Canada).

\section{Ex Vivo Radiography Experiments}

Mice received tail vein injections of $20 \mathrm{MBq}$ of $2-{ }^{18} \mathrm{~F}-\mathrm{FEtOH}$, followed $55 \mathrm{~min}$ later by tail vein injection of $50 \mu \mathrm{L}$ of Hoechst 33342 (10 mg/mL; Thermo Fisher Scientific). Drug-treated mice received either nicotinamide (500 mg/kg; Sigma-Aldrich) $30 \mathrm{~min}$ before $2-{ }^{18} \mathrm{~F}-\mathrm{FEtOH}$, or hydralazine $(10 \mathrm{mg} / \mathrm{kg}$; Sigma-Aldrich) or pentoxifylline $\left(50 \mathrm{mg} / \mathrm{kg}\right.$; Sigma-Aldrich) $15 \mathrm{~min}$ before $2-{ }^{18} \mathrm{~F}-\mathrm{FEtOH}$. Tumors were harvested and immediately embedded in optimalcutting-temperature compound, sectioned onto microscope slides, and incubated on a phosphor screen for 90 min before detection using a Typhoon FLA 9500 scanner (GE Life Sciences).

Phosphor images were analyzed using ImageJ software. Activity histograms were gathered from regions of interest drawn around individual tumors. The line profile function on ImageJ was used to quantify regional differences in $2-{ }^{18} \mathrm{~F}-\mathrm{FEtOH}$ activity. This tool returns the pixel intensity at each point along a line drawn across the full width of each tumor radiography image. To quantify the presence of central ischemia, the intensity of ${ }^{18} \mathrm{~F}$ activity at the center of each tumor was calculated as the average of the 11 pixels closest to the midpoint of each line. Tumor-center intensity values were divided by the maximum intensity value of the entire tumor to express the tumorcenter values as a fraction of the maximum. Three line profiles were drawn and data averaged for each tumor image.

\section{PET Imaging Experiments}

PET imaging was conducted as previously published (21). The rate of irreversible $2-{ }^{18} \mathrm{~F}-\mathrm{FEtOH}$ uptake was determined with the Patlak method (25) using image-derived input functions from the vena cava as previously published (26) and analysis conducted with MatLab software (The MathWorks).

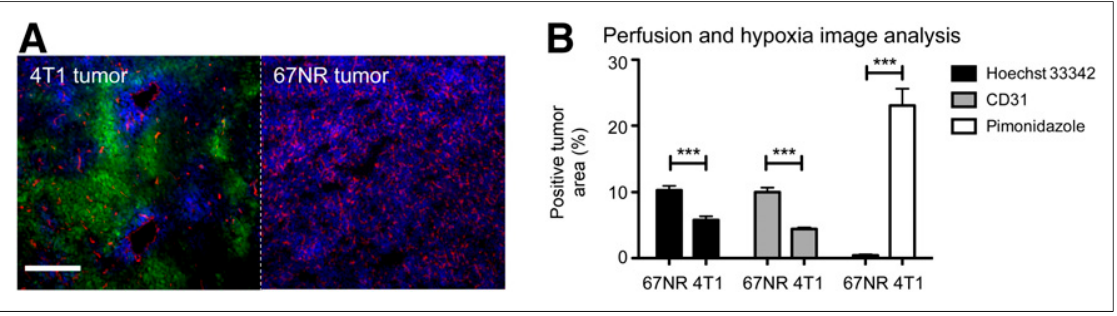

FIGURE 1. (A) Immunofluorescent images of $67 \mathrm{NR}$ and $4 \mathrm{~T} 1$ tumors; perfusion indicated by Hoechst 33342 (blue), vascular endothelium by CD31 (red), and hypoxia by pimonidazole (green). Scale bar $250 \mu \mathrm{m}$. (B) Percentage tumor area positive for Hoechst 33342, CD31, and pimonidazole $\left(n=8\right.$, mean \pm SEM; unpaired $t$ test, $\left.{ }^{\star \star *} P<0.0001\right)$.

\section{RESULTS}

\section{2-18F-FEtOH Uptake Depicts Regional Perfusion Differences Within Tumors}

Immunofluorescent images of 67NR and 4T1 (Fig. 1A) tumors grown orthotopically in contralateral mammary fat pads showed distinct phenotypes of vascular density and blood vessel function. CD31 vascular endothelium staining was significantly more prevalent in 67NR than in 4T1 tumors (Fig. 1B; mean $\pm \mathrm{SEM}, 10.01 \% \pm 0.71 \%$ vs. $4.43 \% \pm$ $0.23 \%$ ), indicating greater vascular density in 67NR tumors. We also assessed uptake of 

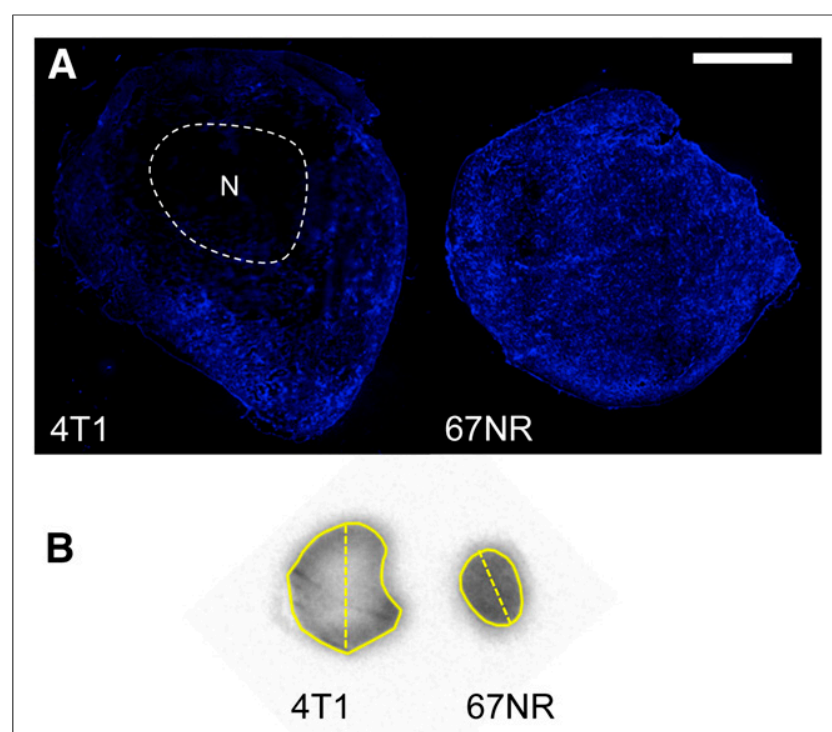

\section{C \\ $2-{ }^{18} \mathrm{~F}-\mathrm{FEtOH}$ at tumor center relative to max intensity}

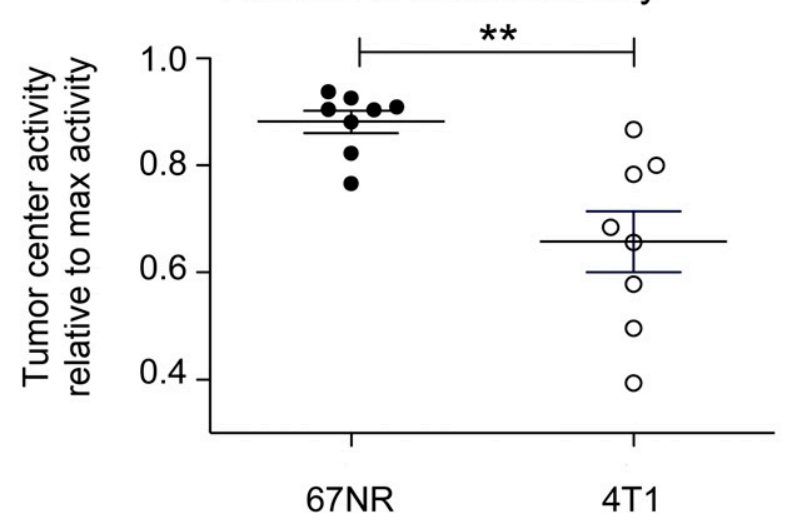

FIGURE 2. (A) Fluorescent microscopy of Hoechst 33342 in 4T1 and 67NR tumors identifies central necrosis $(\mathrm{N})$ in $4 \mathrm{~T} 1$ tumors. Scale bar $1,000 \mu \mathrm{m}$. (B) $2-{ }^{18} \mathrm{~F}-\mathrm{FEtOH}$ radiography of untreated tumors. Solid yellow line defines tumor border; dashed lines are examples of where line profiles are drawn. (C) Tumor-center ${ }^{18} \mathrm{~F}$ intensity expressed relative to tumor-maximum intensity displays low $2{ }^{-18} \mathrm{~F}$-FEtOH uptake in center of 4T1 tumors $\left(n=7 ; t\right.$ test, $\left.{ }^{\star \star} P<0.05\right)$. $\max =$ maximum.

intravenously administered Hoechst 33342 fluorescent dye, which diffuses out of perfused blood vessels to intercalate into cellular DNA. Nearly twice as much tumor area stained positive for Hoechst 33342 in $67 \mathrm{NR}$ versus $4 \mathrm{~T} 1$ tumors (Fig. 1B; $10.30 \% \pm$ $0.65 \%$ vs. $5.79 \% \pm 0.56 \%$ ), indicating that $67 \mathrm{NR}$ tumor blood vessels are relatively well perfused. Consistently, we observed greater staining of the exogenous hypoxia reporter pimonidazole in $4 \mathrm{~T} 1$ tumors than in $67 \mathrm{NR}$ tumors (Fig. $1 \mathrm{~B} ; 23.06 \% \pm 2.55 \%$ vs. $0.45 \% \pm 0.15 \%$ ). Overall the $4 \mathrm{~T} 1$ tumors were found to exhibit low blood vessel density, poor perfusion, and large amounts of hypoxia, which opposes their syngeneic counterpart 67NR tumors and agrees with past research $(27,28)$.

The microenvironment of $4 \mathrm{~T} 1$ tumors provides a model for assessing regional perfusion differences due to the common presence of necrosis in the center of these tumors. The centers of 4T1 tumors are poorly perfused and do not contain viable tissue, as indicated by a lack of Hoechst 33342 staining (Fig. 2A). This is again in contrast to 67NR tumors that display perfusion throughout the tumor (Fig. 2A). We exploited this feature as a first test for $2-{ }^{18} \mathrm{~F}-\mathrm{FEtOH}$ as a reporter of tumor perfusion, hypothesizing that the radiotracer would be present only in the periphery of $4 \mathrm{~T} 1$ tumors and throughout 67NR tumors.

Ex vivo radiography of $2-{ }^{18} \mathrm{~F}-\mathrm{FEtOH}$ conducted on tumors excised 55 min after injection displayed patterns similar to Hoechst 33342; low activity was observed in central regions of 4T1 tumors, moderate activity in the periphery of $4 \mathrm{~T} 1$ tumors, and high activity throughout 67NR tumors (Fig. 2B). To quantify these observations, the pixel intensities were assessed in line profiles drawn across the width of each tumor's radiography image (e.g., Supplemental Fig. 1; supplemental materials are available at http://jnm. snmjournals.org). Values of the 11 pixels closest to the midpoint of the line profile were averaged to represent the $2-{ }^{18} \mathrm{~F}-\mathrm{FEtOH}$ activity in the tumor center. These values were compared with the maximum intensity in the entire associated tumor image. We observed that pixel intensities in the center of 67NR tumors were $88 \% \pm 2.0 \%$ the value of the maximum $67 \mathrm{NR}$ intensity, whereas those in the center of $4 \mathrm{~T} 1$ tumors were only $65 \% \pm 5.7 \%$ of their tumor maximums (Fig. 2C). These results suggest that $2-{ }^{18} \mathrm{~F}-$ FEtOH uptake provides intratumoural resolution of perfusion status that is in agreement with blood vessel density, perfusion status, and hypoxia in 4T1 and 67NR tumors.

\section{2-18FEtOH Uptake Agrees with Validated Perfusion Reporter ${ }^{14} \mathrm{C}$-IAP}

To link $2{ }^{18} \mathrm{~F}-\mathrm{FEtOH}$ uptake with tissue perfusion, we coinjected $2-{ }^{18} \mathrm{~F}$-FEtOH with ${ }^{14} \mathrm{C}$-IAP, which is a validated perfusion reporter used to study cerebral and tumor perfusion (29-31) by measuring ex vivo ${ }^{14} \mathrm{C}$-IAP activity. We found that $2-{ }^{18} \mathrm{~F}-\mathrm{FEtOH}$ and ${ }^{14} \mathrm{C}$-IAP radioactivity in brain tissue and in $67 \mathrm{NR}$ and $4 \mathrm{~T} 1$ tumors provided clear distinction of each tissue type, with a general progression from poorly perfused $4 \mathrm{~T} 1$ tumors through $67 \mathrm{NR}$ tumors and up to well-perfused brain tissue. $2-{ }^{18} \mathrm{~F}-\mathrm{FEtOH}$ and ${ }^{14} \mathrm{C}$-IAP activities were significantly correlated, and the dynamic range of $2-{ }^{18} \mathrm{~F}-\mathrm{FEtOH}$ activity was greater than ${ }^{14} \mathrm{C}$-IAP, with a slope of $1.162 \pm 0.1416$ percentage injected dose per gram $(\% \mathrm{ID} / \mathrm{g})$ across all tissues analyzed (Fig. 3).

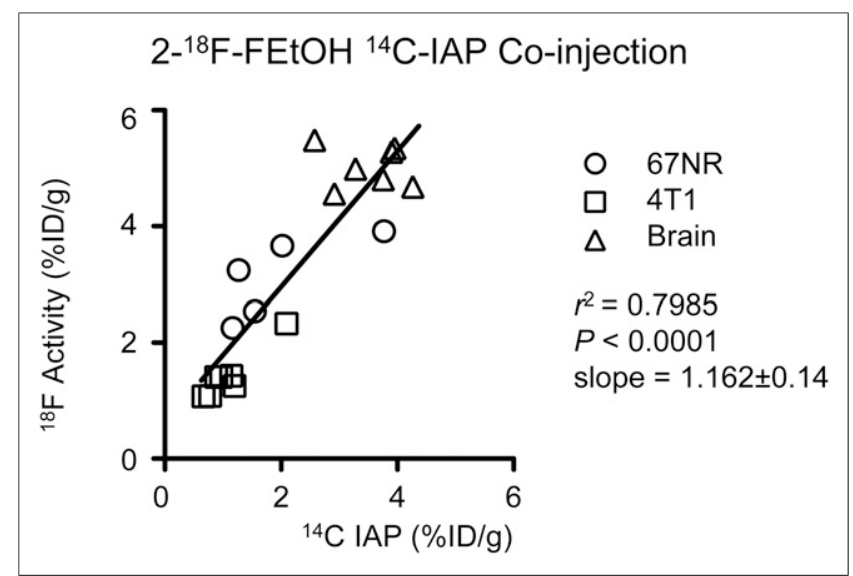

FIGURE 3. $2-{ }^{18} \mathrm{~F}-\mathrm{FEtOH}$ was coinjected with ${ }^{14} \mathrm{C}-\mathrm{IAP}$ and bulk tissue radioactivity measured to determine uptake of each radiotracer. Linear regression trend line across all 3 tissues is displayed in black. 


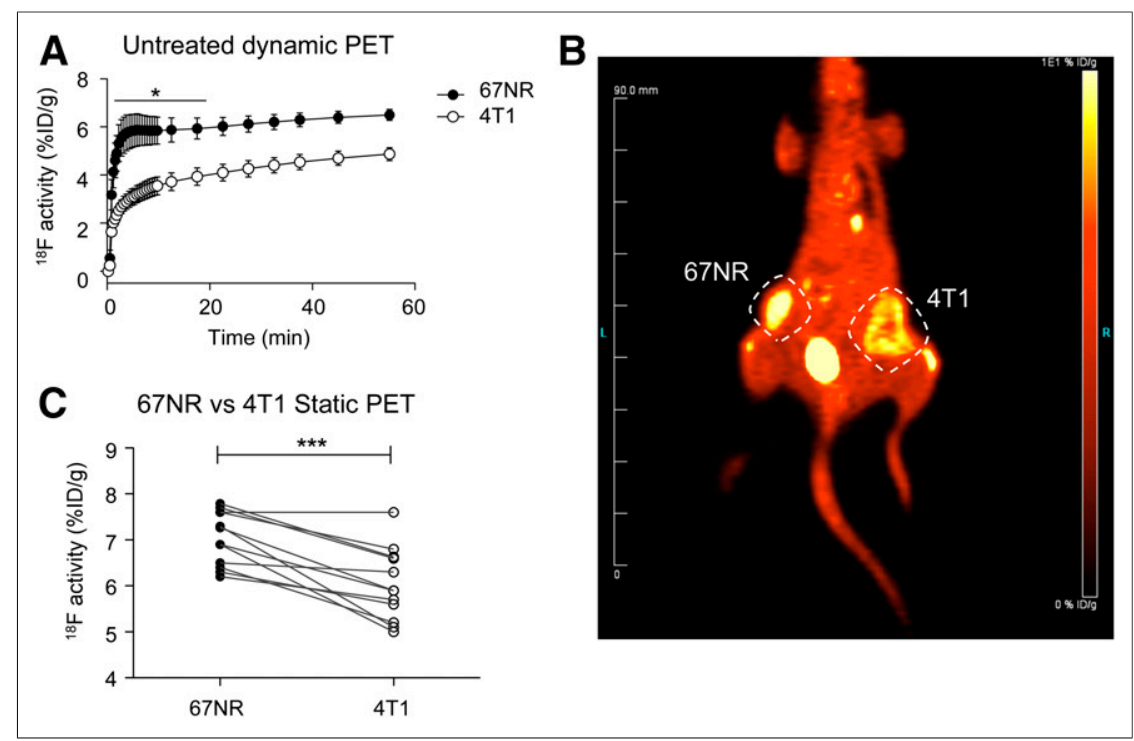

FIGURE 4. $2-{ }^{18} \mathrm{~F}-\mathrm{FEtOH}$ distinguishes $67 \mathrm{NR}$ from $4 \mathrm{~T} 1$ tumors. (A) Mean tumor $2-{ }^{18} \mathrm{~F}-\mathrm{FEtOH}$ activity during dynamic PET $(n=4$, mean \pm SEM, 2-way ANOVA with Bonferroni multiple comparisons, $67 \mathrm{NR}$ vs. $\left.4 \mathrm{~T} 1,{ }^{*} P<0.05\right)$. (B) Sample whole-body static PET image $55 \mathrm{~min}$ after injection. (C) Mean tumor $2-{ }^{18} \mathrm{~F}-\mathrm{FEtOH}$ activity from static PET $\left(n=12\right.$; paired $t$ test, $\left.{ }^{* \star *} P<0.0001\right)$.

allows for the signal stability displayed in Figure 4 , it poses the risk of having $2-{ }^{18} \mathrm{~F}$ FEtOH uptake select for cells and tissues better able to metabolize FEtOH. To address this concern we conducted Patlak modeling (25) of $2-{ }^{18} \mathrm{~F}-\mathrm{FEtOH}$ uptake into $67 \mathrm{NR}$ and $4 \mathrm{~T} 1$ tumors using image-derived input functions (Supplemental Fig. 3) based on voxels of ${ }^{18} \mathrm{~F}$ activity derived from dynamic PET images of the mouse vena cava (26) after injection of $2-{ }^{18} \mathrm{~F}-$ FEtOH. The Patlak method outputs an irreversible uptake rate constant $\mathrm{Ki}$ that is dependent on both metabolism of the radiotracer and its concentration in the plasma. Thus, Ki reflects possible intrinsic differences between cells and the delivery of $2-{ }^{18} \mathrm{~F}-\mathrm{FEtOH}$ through perfused vasculature. When $\mathrm{Ki}$ values were determined on the basis of tissue activity curves of full tumor volumes, the $\mathrm{Ki}$ was significantly greater in $67 \mathrm{NR}$ tumors than $4 \mathrm{~T} 1$ tumors (1.6-fold difference, data not shown). However, regions of interest drawn selectively in well-perfused peripheral $4 \mathrm{~T} 1$ regions pro-

\section{2-18F-FEtOH Provides Distinction Between 67NR and 4T1 Tumors for Extended Time}

We measured $2-{ }^{18} \mathrm{~F}-\mathrm{FEtOH}$ uptake in $67 \mathrm{NR}$ and $4 \mathrm{~T} 1$ tumors for 55 min after injection using dynamic PET imaging. On the basis of previous work showing stable uptake of $2-{ }^{18} \mathrm{~F}-\mathrm{FEtOH}$ into cells (22), we hypothesized that $2-{ }^{18} \mathrm{~F}-\mathrm{FEtOH}$ activity would reach plateaus reflective of tumor perfusion status. In baseline scans, $2{ }^{18} \mathrm{~F}-$ FEtOH uptake into 67NR tumors plateaued at approximately 5.8 $\%$ ID/g after $2 \mathrm{~min}$ and activity did not significantly change over the remaining protocol (Fig. 4A). 2-18 F-FEtOH activity in 4T1 tumors did not reach a plateau until $30 \mathrm{~min}$ after injection at approximately $4.5 \% \mathrm{ID} / \mathrm{g}$, after which no further increase was observed (Fig. 4A). $2-{ }^{18} \mathrm{~F}-\mathrm{FEtOH}$ activity in 67NR tumors was significantly greater than 4T1 from 1 until 12 min after injection (Fig. 4A). We obtained static PET scans 55 min after injection of $2-{ }^{18} \mathrm{~F}-\mathrm{FEtOH}$ to further assess this late time point in mice not anesthetized during tracer uptake (Fig. 4B). Pairwise assessment of mean $2-{ }^{18} \mathrm{~F}-\mathrm{FEtOH}$ activity in each tumor revealed greater activity in 67NR versus the same-mouse 4T1 tumors in 11 of 12 mice (Fig. 4C). Further analysis computed static PET data into cumulative frequency plots (Supplemental Fig. 2A) to calculate the $2-{ }^{18} \mathrm{~F}-\mathrm{FEtOH}$ activity level representative of the 50th and 90th voxel percentiles. Results showed that both the 50th and the 90th percentile activity levels were greater in the 67NR tumors (Supplemental Fig. 2B; mean \pm SEM, $7.75 \pm 0.18$ vs. $6.58 \pm 0.31$ $\% \mathrm{ID} / \mathrm{g}$ and $9.29 \pm 0.19$ vs. $7.71 \pm 0.23 \% \mathrm{ID} / \mathrm{g}, P<0.05)$. These data confirm that the $2-{ }^{18} \mathrm{~F}-\mathrm{FEtOH}$ radiotracer successfully distinguishes 67NR and 4T1 tumors using dynamic PET imaging or static PET imaging, including the potential for $2-{ }^{18} \mathrm{~F}-\mathrm{FEtOH}$ to report perfusion for a longer time compared with previously published PET-based perfusion reporters.

\section{Irreversible Uptake Rate of $2-{ }^{18}$ F-FEtOH Is Dependent on Perfusion and Not Tumor Line}

We hypothesized that $2-{ }^{18} \mathrm{~F}-\mathrm{FEtOH}$ is irreversibly taken up into cells, fitting with a 2-tissue-compartment model. Although this duced $\mathrm{Ki}$ values similar to those in whole 67NR tumors (Fig. 5). In contrast, the poorly perfused center $4 \mathrm{~T} 1$ regions possessed $\mathrm{Ki}$ values that were significantly lower than $67 \mathrm{NR}$ tumors or peripheral regions of $4 \mathrm{~T} 1$ tumors. These data support that differences in $2-{ }^{18} \mathrm{~F}-$ FEtOH uptake are due to perfusion differences and not differences in FEtOH metabolism.

\section{2-18F-FEtOH Uptake Into Tumors Is Modified by Perfusion-Modifying Drugs}

We next tested 3 blood flow-modifying agents to observe whether $2-{ }^{18} \mathrm{~F}-\mathrm{FEtOH}$ uptake into $4 \mathrm{~T} 1$ and $67 \mathrm{NR}$ tumors would

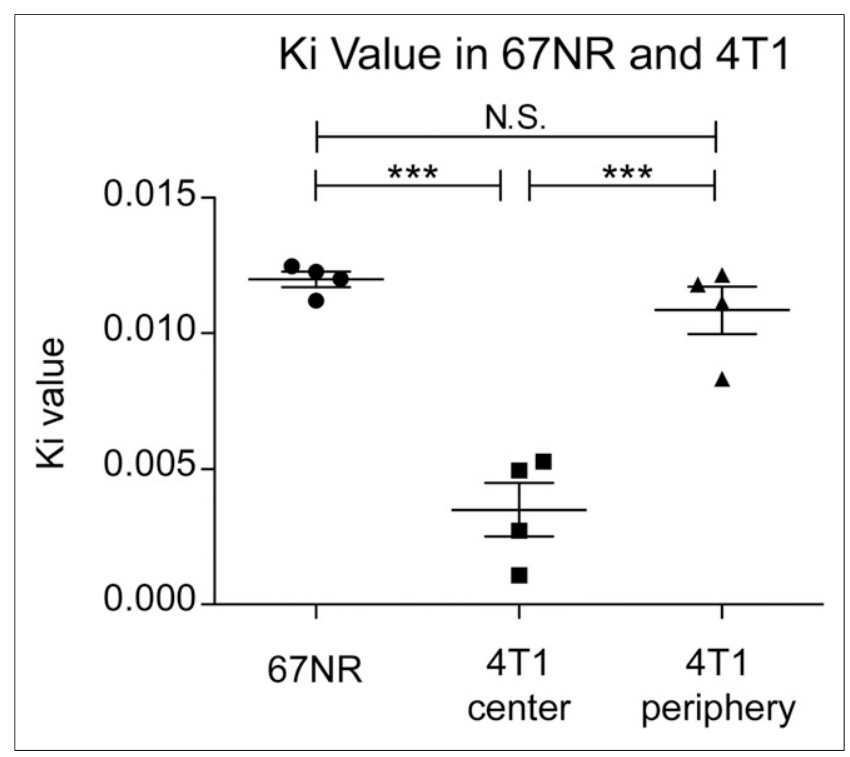

FIGURE 5. Patlak irreversible rate constant Ki was computed for whole 67NR tumor volumes and compared with select regions of $4 \mathrm{~T} 1$, poorly perfused tumor center, and well-perfused periphery $(n=4$; 1-way ANOVA with Tukey multiple-comparisons test, $\left.{ }^{\star \star \star} P<0.0001\right)$. N.S. = not significant. 


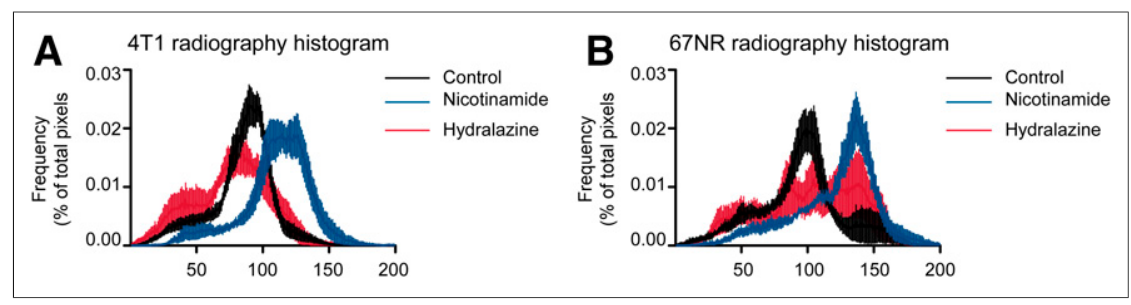

FIGURE 6. Perfusion-modifying drugs alter $2-{ }^{-18} \mathrm{~F}-\mathrm{FEtOH}$ uptake into $4 \mathrm{~T} 1$ (A) and 67NR (B) tumors. Mice were administered either nicotinamide or hydralazine before $2-{ }^{18} \mathrm{~F}-\mathrm{FEtOH}$ injection. Radioactivity was detected using radiography and expressed as histograms $(n=5$, mean \pm SEM).

reflect the perfusion changes. We used 2 agents to increase tumor blood flow, nicotinamide (32) and pentoxifylline (33), and used hydralazine as an agent to reduce tumor blood flow (34) before ex vivo radiography of $2-{ }^{18} \mathrm{~F}-\mathrm{FEtOH}$ uptake from tumors harvested $55 \mathrm{~min}$ after radiotracer injection. Nicotinamide increased $2-{ }^{18} \mathrm{~F}-$ FEtOH uptake in both $67 \mathrm{NR}$ and $4 \mathrm{~T} 1$ tumors, indicated by the rightward shifts toward greater pixel intensity on histograms (Figs. $6 \mathrm{~A}$ and $6 \mathrm{~B})$. This is consistent with the known activity of nicotinamide to improve solid tumor perfusion. Similarly, pentoxifylline increased $2-{ }^{18} \mathrm{~F}-\mathrm{FEtOH}$ uptake in both tumors (Supplemental Figs. $4 \mathrm{~A}$ and $4 \mathrm{~B}$ ), with a more modest rightward shift compared with nicotinamide for either tumor type. Hydralazine induced a leftward shift in 4T1 tumor histograms (Fig. 6A), indicating a reduction in perfusion that is consistent with the known activity of hydralazine. However, 67NR tumors did not display a reliable shift in response to hydralazine at this time point when tumors were harvested 55 min after $2-{ }^{18} \mathrm{~F}-\mathrm{FEtOH}$ injection (Fig. 6B).

To further investigate the responses of 67NR and 4T1 tumors to hydralazine, we performed dynamic PET imaging of $2-{ }^{18} \mathrm{~F}-\mathrm{FEtOH}$ uptake after hydralazine administration compared with baseline images (sample images in Supplemental Fig. 5). 67NR tumors treated with hydralazine displayed a significant reduction in $2-{ }^{18} \mathrm{~F}-\mathrm{FEtOH}$ activity over the first $10 \mathrm{~min}$ of dynamic PET imaging (Fig. 7A). 67NR 2-18 F-FEtOH activity in control and hydralazine groups converged within $55 \mathrm{~min}$, agreeing with the ex vivo radiography data (Fig. 6B). The mean $2-{ }^{18} \mathrm{~F}-\mathrm{FEtOH}$ activity in 4T1 tumors was reduced throughout the dynamic scan (Fig. 7B). These data indicate that dynamic imaging is required to accurately assess the changes in tumor perfusion induced by blood flow-modifying drugs.

\section{DISCUSSION}

This study presents $2-{ }^{18} \mathrm{~F}-\mathrm{FEtOH}$ as a novel radiotracer for measuring tumor perfusion. We have found that uptake of $2-{ }^{18} \mathrm{~F}-\mathrm{FEtOH}$ is dependent on regional perfusion status, with $2-{ }^{18} \mathrm{~F}-\mathrm{FEtOH}$ uptake in agreement with the intratumoural heterogeneity of Hoechst 33342 staining (Fig. 2). 2-18 F-FEtOH uptake also accurately reflected the perfusion statuses of 2 tumor models with distinct vascular and hypoxic phenotypes (Figs. 1-4). 2-18 F-FEtOH uptake into 67NR and 4T1 tumors and brain tissue displayed strong agreement with the validated perfusion reporter ${ }^{14} \mathrm{C}$-IAP, whereas $2-{ }^{18} \mathrm{~F}-\mathrm{FEtOH}$ uptake displayed a greater dynamic range in distinguishing each tissue (Fig. 3).

We also showed that $2-{ }^{18} \mathrm{~F}-\mathrm{FEtOH}$ uptake can be used to measure the effects of pharmacologic agents that modify tumor perfusion (Figs. 6 and 7). We tested 2 vasoactive drugs (nicotinamide and hydralazine) as well as pentoxifylline, which increases perfusion by improving the flexibility of red blood cells to facilitate their flow through tortuous tumor vessels $(35,36)$. Further experiments with hydralazine showed that assessing the effects of perfusion-modifying agents is aided by time course analyses such as dynamic PET imaging (Fig. 7). Taken together, our data show the potential for $2-{ }^{18} \mathrm{~F}-\mathrm{FEtOH}$ uptake quantified by dynamic PET imaging to be used to screen blood flow-modifying agents for use as therapeutic adjuvants to existing cancer therapies.

The extended half-life of the ${ }^{18} \mathrm{~F}$ isotope provides ${ }_{2-}{ }^{18} \mathrm{~F}-\mathrm{FEtOH}$ with the benefits of accessibility and eased protocol timelines compared with ${ }^{15} \mathrm{O}$ radiotracers. $2-{ }^{18} \mathrm{~F}-\mathrm{FEtOH}$ is also stably taken up into cells and produces an activity plateau in well-perfused tissue as measured previously in many healthy tissues (21). An extended half-life and activity plateau provides a unique opportunity to detect perfusion at later time points after injection, with activity plateaus lasting up to $30 \mathrm{~min}$ and later time points exhibiting only a small increase in activity (Figs. 4 and 7).

The data displayed in this study present the utility of $2-{ }^{18} \mathrm{~F}$ FEtOH as a general indicator of tumor perfusion, distinguishing well-perfused and poorly perfused regions. To support that perfusion is the driving factor in $2-{ }^{18} \mathrm{~F}-\mathrm{FEtOH}$ uptake in this study and not cellular differences in metabolic trapping, we applied the Patlak method to determine the irreversible uptake rate constant $\mathrm{Ki}$. The value of $\mathrm{Ki}$ is reflective of both the concentration of $2-{ }^{18} \mathrm{~F}$ FEtOH delivered to a tissue and the ability for the tissue to metabolize the radiotracer into cell impermeable metabolites. In this study, Ki values did not vary between 67NR tumors and well-perfused regions of 4T1 tumors in the same animal (Fig. 5). Rather, Ki values varied strongly within 4T1 tumors in a regional manner, with the lowest $\mathrm{Ki}$ values associated with $4 \mathrm{~T} 1 \mathrm{tu}-$ mor centers (Fig. 5), which we also showed to be poorly perfused (Fig. 2). These modeling data are somewhat limited by the use of an image-derived input function instead of direct blood draws to assess $2-{ }^{18} \mathrm{~F}$ FEtOH in the bloodstream, although image analysis methods have fared well when directly compared with blood collection methods (26). Future kinetic modeling of
FIGURE 7. Hydralazine slows $2-{ }^{18} \mathrm{~F}-\mathrm{FEtOH}$ delivery. ${ }^{2-}{ }^{18} \mathrm{~F}-\mathrm{FEtOH}$ mean activity over dynamic PET comparing baseline $(\bullet)$ scans and hydralazine $(\circ)$ scans for $67 \mathrm{NR}(\mathrm{A})$ and $4 \mathrm{~T} 1$ (B) $(n=4$, mean \pm SEM; Bonferroni multiple comparisons, $\left.{ }^{\star} P<0.05\right)$. 
$2-{ }^{18} \mathrm{~F}-\mathrm{FEtOH}$ should include analysis of FEtOH metabolites and assessment of enzymes that will relate to enhanced metabolic trapping.

Future studies should evaluate $2-{ }^{18} \mathrm{~F}-\mathrm{FEtOH}$ uptake as a measure of tumor perfusion in other solid tumor types. Of additional interest will be investigating the oxygenation of tumor regions with low $2-{ }^{18} \mathrm{~F}-\mathrm{FEtOH}$ activity to distinguish between poorly perfused and poorly oxygenated tumor regions. Furthermore, a comparison of $2-{ }^{18} \mathrm{~F}-\mathrm{FEtOH}$ perfusion and PET-based hypoxia measurements to predict patient response to radiation therapy and particularly chemotherapy, for which other perfusion reporters have provided strong prognostic data on tumor response, is of interest. The accessibility benefit of $2-{ }^{18} \mathrm{~F}-\mathrm{FEtOH}$ over ${ }^{15} \mathrm{O}$ perfusion reporters and the ability to image $2-{ }^{18} \mathrm{~F}-\mathrm{FEtOH} 55 \mathrm{~min}$ after injection would also make $2-{ }^{18} \mathrm{~F}$ $\mathrm{FEtOH}$ an appealing candidate to monitor tumor response during therapy to determine how tumor perfusion responds to therapy. Previous work showing $2-{ }^{18} \mathrm{~F}-\mathrm{FEtOH}$ uptake in normal tissues (21) suggests that $2-{ }^{18} \mathrm{~F}-\mathrm{FEtOH}-$ based perfusion measurements may have applicability beyond solid tumors, with a potential for measuring tissue perfusion in other disease states. Taken together, our data support the further development and application of $2-{ }^{18} \mathrm{~F}-\mathrm{FEtOH}$ as a PET-based marker of tissue and solid tumor perfusion.

\section{CONCLUSION}

$2-{ }^{18} \mathrm{~F}-\mathrm{FEtOH}$ is a novel PET reporter of perfusion. ${ }^{2-{ }^{18} \mathrm{~F}-}$ FEtOH displays intratumour resolution of perfusion, discriminates well-perfused and poorly perfused tumors, responds to tumor perfusion-modifying pharmaceuticals, and provides an extended window of detection compared with current PET perfusion reporters.

\section{DISCLOSURE}

This work was supported by the Canadian Institutes of Health Research (CIHR; MOP-126138) and the BC Cancer Foundation. Brennan J. Wadsworth was supported by a National Sciences and Engineering Research Council of Canada Scholarship and by Jean MacDonald and Northern Telecom Graduate Fellowships. Brennan J. Wadsworth is a Scholar in The Terry Fox Foundation initiative for Strategic Training in Transdisciplinary Radiation Science for the 21st Century (STARS21). Kevin L. Bennewith is a Michael Smith Foundation for Health Research Biomedical Research Scholar. No other potential conflict of interest relevant to this article was reported.

\section{REFERENCES}

1. Vordermark D, Horsman MR. Hypoxia as a biomarker and for personalized radiation oncology. Recent Results Cancer Res. 2016;198:123-142.

2. Bennewith KL, Dedhar S. Targeting hypoxic tumour cells to overcome metastasis. BMC Cancer. 2011;11:504.

3. Hirata K, Terasaka S, Shiga T, et al. ${ }^{18} \mathrm{~F}$-fluoromisonidazole positron emission tomography may differentiate glioblastoma multiforme from less malignant gliomas. Eur J Nucl Med Mol Imaging. 2012;39:760-770.

4. Cheng J, Lei L, Xu J, et al. ${ }^{18} \mathrm{~F}$-fluoromisonidazole PET/CT: a potential tool for predicting primary endocrine therapy resistance in breast cancer. $\mathrm{J} \mathrm{Nucl} \mathrm{Med}$. 2013;54:333-340.

5. Dewhirst MW, Cao Y, Moeller B. Cycling hypoxia and free radicals regulate angiogenesis and radiotherapy response. Nat Rev Cancer. 2008;8:425-437.

6. Bruehlmeier M, Roelcke U, Schubiger PA. Assessment of hypoxia and perfusion in human brain tumors using PET with ${ }^{18} \mathrm{~F}$-fluoromisonidazole and ${ }^{15} \mathrm{O}-\mathrm{H} 2 \mathrm{O} . J$ Nucl Med. 2004;45:1851-1859.

7. Lehtiö K, Oikonen V, Grönroos T, et al. Imaging of blood flow and hypoxia in head and neck cancer: initial evaluation with $\left[{ }^{15} \mathrm{O}\right] \mathrm{H}_{2} \mathrm{O}$ and $\left[{ }^{18} \mathrm{~F}\right]$ fluoroerythronitroimidazole PET. J Nucl Med. 2001;42:1643-1652.

8. Dunnwald LK, Gralow JR, Ellis GK, et al. Tumor metabolism and blood flow changes by positron emission tomography: relation to survival in patients treated with neoadjuvant chemotherapy for locally advanced breast cancer. J Clin Oncol. 2008;26:4449-4457.
9. Humbert O, Riedinger J-MM, Vrigneaud J-MM, et al. ${ }^{18}$ F-FDG PET derived tumor blood flow changes after one cycle of neoadjuvant chemotherapy predicts outcome in triple-negative breast cancer. J Nucl Med. 2016;57:1707-1712.

10. Bernstein JM, Kershaw LE, Withey SB, et al. Tumor plasma flow determined by dynamic contrast-enhanced MRI predicts response to induction chemotherapy in head and neck cancer. Oral Oncol. 2015;51:508-513.

11. Huang Z, Yuh KA, Lo SS, et al. Validation of optimal DCE-MRI perfusion threshold to classify at-risk tumor imaging voxels in heterogeneous cervical cancer for outcome prediction. Magn Reson Imaging. 2014;32:1198-1205.

12. Sorensen AG, Emblem KE, Polaskova $P$, et al. Increased survival of glioblastoma patients who respond to antiangiogenic therapy with elevated blood perfusion. Cancer Res. 2012;72:402-407.

13. Herrero P, Markham J, Bergmann SR. Quantitation of myocardial blood flow with $\mathrm{H} 2{ }^{15} \mathrm{O}$ and positron emission tomography: assessment and error analysis of a mathematical approach. J Comput Assist Tomogr. 1989;13:862-873.

14. Bergmann SR, Fox KA, Rand AL, McElvany KD. Quantification of regional myocardial blood flow in vivo with H215O. Circulation. 1984;70:724-733.

15. Frackowiak RS, Lenzi GL, Jones T, Heather JD. Quantitative measurement of regional cerebral blood flow and oxygen metabolism in man using ${ }^{15} \mathrm{O}$ and positron emission tomography: theory, procedure, and normal values. J Comput Assist Tomogr. 1980;4:727-736.

16. Berridge MS, Adler LP, Nelson AD, et al. Measurement of human cerebral blood flow with [ $\left[{ }^{15} \mathrm{O}\right]$ butanol and positron emission tomography. J Cereb Blood Flow Metab. 1991;11:707-715.

17. Schelbert HR, Phelps ME, Huang SC, et al. N-13 ammonia as an indicator of myocardial blood flow. Circulation. 1981;63:1259-1272.

18. Huang SC, Williams BA, Krivokapich J, Araujo L, Phelps ME, Schelbert HR. Rabbit myocardial ${ }^{82} \mathrm{Rb}$ kinetics and a compartmental model for blood flow estimation. Am J Physiol. 1989;256:H1156-H1164.

19. Cal-González J, Herraiz JL, España S, Desco M, Vaquero J, Udías J. Positron range effects in high resolution 3D PET imaging. IEEE Nucl Sci Symp Conf Rec. 2009;2788-2791.

20. Maddahi J. Properties of an ideal PET perfusion tracer: new PET tracer cases and data. J Nucl Cardiol. 2012;19:S30-S37.

21. Pan J, Pourghiasian M, Hundal N, et al. f- $\left[{ }^{18} \mathrm{~F}\right]$ fluoroethanol and $3-\left[{ }^{18} \mathrm{~F}\right]$ fluoropropanol: facile preparation, biodistribution in mice, and their application as nucleophiles in the synthesis of $\left[{ }^{18} \mathrm{~F}\right]$ fluoroalkyl aryl ester and ether PET tracers. Nucl Med Biol. 2013;40:850-857.

22. Tewson TJ, Welch MJ. Preparation and preliminary biodistribution of "no carrier added" fluorine1-8 fluoroethanol. J Nucl Med. 1980;21:559-564.

23. Aslakson CJ. Selective events in the metastatic process defined by analysis of the sequential dissemination of subpopulations of a mouse mammary tumor. Cancer Res. 1992;52:1399-1405.

24. Horton RW, Pedley TA, Meldrum BS. Regional cerebral blood flow in the rat as determined by particle distribution and by diffusible tracer. Stroke. 1980;11:39-44.

25. Patlak CS, Blasberg RG. Graphical evaluation of blood-to-brain transfer constants from multiple-time uptake data. Generalizations. J Cereb Blood Flow Metab. 1985;5:584-590.

26. Lanz B, Poitry-Yamate C, Gruetter R. Image-derived input function from the vena cava for ${ }^{18}$ F-FDG PET studies in rats and mice. J Nucl Med. 2014;55:1380-1388.

27. Dexter DL, Kowalski HM, Blazar BA, Fligiel Z, Vogel R. Heterogeneity of tumor cells from a single mouse mammary tumor. Cancer Res. 1978;38:3174-3181.

28. Lou Y, McDonald P, Oloumi A, et al. Targeting tumor hypoxia: suppression of breast tumor growth and metastasis by novel carbonic anhydrase IX inhibitors. Cancer Res. 2011;71:3364-3376.

29. Wang Z, Stefanko D, Guo Y, et al. Evidence of functional brain reorganization on the basis of blood flow changes in the CAG140 knock-in mouse model of Huntington's disease. Neuroreport. 2016;27:632-639.

30. Tozer GM, Shaffi KM. Modification of tumour blood flow using the hypertensive agent, angiotensin II. Br J Cancer. 1993;67:981-988.

31. Tatsumi M, Yutani K, Kusuoka H, Nishimura T. Technetium-99m HL91 uptake as a tumour hypoxia marker: relationship to tumour blood flow. Eur J Nucl Med. 1999;26:91-94.

32. Chaplin DJ, Horsman MR, Trotter MJ. Effect of nicotinamide on the microregional heterogeneity of oxygen delivery within a murine tumor. $J$ Natl Cancer Inst. 1990;82:672-676.

33. Bennewith KL, Durand RE. Drug-induced alterations in tumour perfusion yield increases in tumour cell radiosensitivity. Br J Cancer. 2001;85:1577-1584.

34. Ljungkvist AS, Bussink J, Rijken P, Raleigh J, Denekamp J, Kogel A. Changes in tumor hypoxia measured with a double hypoxic marker technique. Int $J$ Radiat Oncol Biol Phys. 2000;48:1529-1538.

35. Lee I, Levitt SH, Song CW. Improved tumour oxygenation and radiosensitization by combination with nicotinamide and pentoxifylline. Int J Radiat Biol. 1993;64:237-244.

36. Ehrly AM. The effect of pentoxifylline on the flow properties of human blood. Curr Med Res Opin. 1978;5:608-613. 